\title{
Task-dependent influence of genetic architecture and mating frequency on division of labour in social insect societies
}

\author{
Danesh Tarapore · Dario Floreano · Laurent Keller
}

Received: 25 August 2009 / Revised: 6 November 2009 / Accepted: 11 November 2009 / Published online: 25 November 2009

(C) Springer-Verlag 2009

\begin{abstract}
Division of labour is one of the most prominent features of social insects. The efficient allocation of individuals to different tasks requires dynamic adjustment in response to environmental perturbations. Theoretical models suggest that the colony-level flexibility in responding to external changes and internal perturbation may depend on the within-colony genetic diversity, which is affected by the number of breeding individuals. However, these models have not considered the genetic architecture underlying the propensity of workers to perform the various tasks. Here, we investigated how both within-colony genetic variability (stemming from variation in the number of matings by queens) and the number of genes influencing the stimulus (threshold) for a given task at which workers begin
\end{abstract}

Communicated by M. Beekman

Electronic supplementary material The online version of this article (doi: 10.1007/s00265-009-0885-4) contains supplementary material, which is available to authorized users.

D. Tarapore $\cdot$ L. Keller

Department of Ecology and Evolution (DEE), Biophore,

University of Lausanne (UNIL), Dorigny,

1015 Lausanne, Switzerland

D. Tarapore $(\varangle) \cdot$ D. Floreano

Laboratory of Intelligent Systems (LIS),

Ecole Polytechnique Fédérale de Lausanne (EPFL),

EPFL-STI-IMT-LIS, Station 11, 1015 Lausanne, Switzerland

e-mail: danesh.tarapore@epfl.ch to perform that task jointly influence task allocation efficiency. We used a numerical agent-based model to investigate the situation where workers had to perform either a regulatory task or a foraging task. One hundred generations of artificial selection in populations consisting of 500 colonies revealed that an increased number of matings always improved colony performance, whatever the number of loci encoding the thresholds of the regulatory and foraging tasks. However, the beneficial effect of additional matings was particularly important when the genetic architecture of queens comprised one or a few genes for the foraging task's threshold. By contrast, a higher number of genes encoding the foraging task reduced colony performance with the detrimental effect being stronger when queens had mated with several males. Finally, the number of genes encoding the threshold for the regulatory task only had a minor effect on colony performance. Overall, our numerical experiments support the importance of mating frequency on efficiency of division of labour and also reveal complex interactions between the number of matings and genetic architecture.

Keywords Task allocation - Response thresholds • Number of matings $\cdot$ Number of loci .

Genetic diversity

\section{Introduction}

Ants and other social insects have been extremely successful during the course of evolution, as shown by the sheer magnitude of their numbers and their biomass and energy consumption, which exceed that of 
vertebrates in most terrestrial habitats (Wilson 1975). Central to their ecological success are the high levels of cooperation and sophisticated division of labour (Hölldobler and Wilson 1990). In a colony, there are a number of tasks to attend to such as feeding the brood, foraging for resources, maintaining the nest, and defending the colony. Efficient allocation of individuals to these different tasks requires dynamic adjustments in response to internal perturbations (e.g. due to the mortality of individuals that were specialised in a specific task) or external changes (e.g. intra- and interspecific competition or amount of food available) (Calabi and Traniello 1989; Cerdá et al. 2009; Gordon 1989, 1991; Mercier and Lenoir 1999; Robinson 1992; Seeley 1989; Wilson 1984; Crosland and Traniello 1997; Karsai and Wenzel 2000).

Considerable work has focussed on identifying the mechanisms allowing colonies to efficiently respond to information that exceeds the sensory range or cognitive capacity of any given individual. The large majority of these models are built on the observation that individuals in a colony vary in their propensity to perform different tasks (Jones et al. 2004; Robinson 1992; O'Donnell 1996). In these models, individuals vary intrinsically in the stimulus (threshold) for a given task at which they begin to perform that task. Because individuals with a low threshold for a given stimulus are more likely to perform that task than individuals with a high threshold, intra-colony variation in individual thresholds results in colony division of labour.

An important assumption of most of these models is that the distribution of response thresholds in the group is normally distributed (Graham et al. 2006; Jeanson et al. 2007; Myerscough and Oldroyd 2004). However, very little is known about the genetic architecture of response thresholds. Studies in the honeybee suggest that genotypic variation in tasks may involve a few major loci (Hunt et al. 1995; Page et al. 2000; Rüppell et al. 2004). In many species, a genetic component in response thresholds has also been demonstrated with individuals from different patrilines or matrilines having different propensities to perform certain tasks (Costa and Ross 2003; Frumhoff and Baker 1988; Jones et al. 2004; Julian and Cahan 1999; Oldroyd et al. 1994; Snyder 1992; Lenoir et al. 2006). However, a better understanding of division of labour requires determining how genotypic variation relates to differences in intracolony differences in response thresholds. In a first move toward this goal, Bertram et al. (2003) developed a model where they investigated the effects of several variables, such as the number of genes (loci) and alleles per locus controlling individual response threshold, allele frequencies within the colony and the level of variation in phenotype generated by random environmental effects. Their simulations showed that all these variables had important effects on task allocation and colony behaviour. Similarly, using experimental evolution, Waibel et al. (2006) also showed that the performance of colonies is strongly influenced by the genetic architectures responsible for within-colony variation in response thresholds. However, these two studies did not investigate possible interactions between the level of within-colony genetic diversity resulting from variation in queen mating frequency and/or the number of reproductive queens per colony, and the genetic architecture of response thresholds. This is a fundamental question because there is considerable intra- and interspecific variation in within-colony genetic diversity (Boomsma and Ratnieks 1996; Hughes et al. 2008; Keller and Reeve 1994; Strassmann 2001; Trontti et al. 2007) and the degree of within-colony genetic diversity has been proposed to be a key factor influencing the efficiency of division of labour and, thus, overall colony performance (Fewell and Bertram 1999; Jones et al. 2004; Oldroyd et al. 1993; Ranger and O'Donnell 1999).

In this study, we investigate how the level of withincolony genetic diversity and genetic architecture jointly influence task allocation efficiency and division of labour. We used a probabilistic agent-based simulator (Perez-Uribe et al. 2003) to model a situation where workers had to perform two distinct tasks. The first was a regulatory task where workers had to maintain the amount of a given food item in their colony within predefined bounds. This would, for example, be the case of a honey bee colony maintaining about one kilogram of pollen in the hive or workers regulating within-hive temperature (Seeley 1995). The other was a foraging task where workers had to collect the highest possible amount of a second type of food item. This may, for example, be the case of a honey bee colony maximising the amount of nectar foraged or workers minimising the diseased brood in the hive. The fitness of colonies was a function of workers being able to perform both tasks efficiently. To study the effect of within-colony genetic diversity, we compared the fitness of queens mated with either one, three, five, seven or ten males. To investigate the role of the genetic architecture of the behavioural threshold, we considered the situations where the response threshold of the foraging and regulatory tasks were each under the control of one, three, five, seven or ten independent loci with the same effect. There were thus $125(5 \times 5 \times 5)$ experimental conditions. For each condition, we conducted experimental evolution in ten independent replicates over 100 generations. 


\section{Materials and methods}

\section{Colony tasks}

We conducted 100 generations of artificial selection in populations consisting of 500 colonies. Each colony consisted of one queen and 1,000 workers placed in an environment with an infinite number of two types of food items. Our aim was to mimic a situation where the performance of colonies was a direct function of workers simultaneously performing both the regulatory and the foraging tasks. Thus, the fitness function directly depended on the number of items of the foraging task collected, but these were counted only when the number of food items of the regulatory task was within predefined bounds (140-160 items per colony). The colony lifespan was divided into 100 time-steps. Before evaluating the colony, there were no items of the foraging and regulatory tasks in the nest. The total colony performance $F$ was calculated by adding the performance obtained at each time-step, with $F=\sum_{t=1}^{100} F_{t}$, where colony performance at each time-step $\left(F_{t}\right)$ was quantified as the number of items of the foraging task collected when the number of items of the regulatory task present in the nest was between 140 and 160:

$F_{t}=w_{t} * R_{t}^{\text {for }}$,

where $w_{t}=1$ if $140 \leq R_{t}^{\text {reg }} \leq 160$ and $w_{t}=0$ otherwise.

$R_{t}^{\text {for }}$ represents the number of resources foraged at time-step $t$ and $R_{t}^{\text {reg }}$ the number of items being regulated within the nest at time-step $t$. Thus, if colonies performed well in only one of the two tasks, their fitness was low. This mimics the situations of two vital tasks such as regulation of nest temperature and foraging. If the colony is efficient for foraging but does not regulate nest temperature well, all the brood may die. Inversely, if nest temperature is well controlled, but little food is collected, only few offspring can be reared. The fitness function used assumes a sharp on-off transition on the effects of the foraging behaviour with respect to the regulatory conditions. It is likely that the interaction between tasks is not an absolute on-off switch in nature, but this should not qualitatively affect the result of the simulations.

Worker task allocation followed the response threshold model (Bonabeau et al. 1996; Page and Mitchell 1998). At the beginning of each time-step, a worker had two task stimuli, one for foraging resources and the other for regulating resources. Based on the threshold model, the intensity of the stimulus for each task was inversely proportional to the number of corresponding resources in the nest. Each worker performed the task corresponding to the largest difference between the stimulus and its own response threshold or randomly performed one of the two tasks when the two differences were equal. When both thresholds were higher than the corresponding stimuli, workers remained idle.

During each time-step, a worker had a probability of 0.1 to successfully collect an item corresponding to the task performed and, at each time-step, the number of foraged and regulated resources in the nest were depleted by ten with a probability of 0.5 .

\section{Genetic architecture and mating frequency}

To mimic the haplodiploid genetic system of ants and other social Hymenoptera, we assumed that females (queens and workers) were diploid and males haploid. The response thresholds for the two tasks were each determined by a set of unlinked loci (one, three, five, seven or ten loci per task) with the allelic values at each locus ranging from 0 to 1 (256 possible values). The response threshold of each task was equal to the average of all allelic values at the loci corresponding to the task. Colonies were headed by one queen mated with either one, three, five, seven or ten males. There were, thus, 125 treatments $(5 \times 5$ combinations of genetic architecture for the two tasks and five mating frequencies).

\section{Reproduction and selection}

Artificial selection was conducted in ten independent replicates. At the first generation, the alleles of all 500 queens and their corresponding mates were set randomly to one of the 256 values between 0 and 1 with a resolution of 0.004 . The genotype of each of the 1,000 colony workers was constructed by selecting one of the two alleles at the corresponding maternal locus and the other allele in the queen mate (when queens were multiply mated, we randomly selected one male for each worker produced).

To construct the 500 colonies of the following generation, we selected the 150 colonies with the highest fitness and used fitness proportional selection (i.e. the probability of a colony to contribute to male and queen production was proportional to its fitness) to generate from the 150 colonies the 500 new queens and corresponding number of new males of the next generation. The genotype of the new queen was produced in the same manner as that of the workers, while the genotype of males was simply constructed by randomly selecting one of the maternal alleles for each corresponding locus. The alleles of the new queens and males were 
mutated by adding a random value in the range from -0.1 to +0.1 with a resolution of 0.004 . The values of mutations were generated using a Gaussian distribution so that the magnitude of the value was inversely proportional to its probability of occurrence. Because the workers were sterile and could not transmit their genome, they were not subjected to mutations. For each of the 500 new queens, we randomly selected the corresponding number of mates from the pool of newly created males.

Worker response threshold diversity index and statistical analysis

To investigate how the level of within-colony genetic diversity and the genetic architecture influenced task allocation efficiency, we computed for both the foraging and regulatory tasks a within-colony diversity index. We defined the diversity index as the mean Euclidean distance between response thresholds of all possible pairs of workers within the colony.

To compare the diversity indices between treatments, we averaged, for each treatment and replicate, the diversity index values over the 500 colonies (ten replicates per treatment) at generation 100 . The same procedure was used to compare the colony performance, number of resources foraged and proportion of time colonies maintained the number of resources in the nest within predefined bounds. Statistical significance was determined with ANOVA tests, followed by Tukey-Kramer's multi-comparison tests (Sokal and Rohlf 1995).

To illustrate the effect on colony performance of the number of matings, number of loci encoding the threshold of the foraging task and the number of loci encoding the threshold of the regulatory task, we present the effect of each of these three variables for the extreme values of the other two variables (i.e., one and ten matings, one and ten loci for the foraging task or one and ten loci for the regulatory task). The same procedure was used to illustrate the effect of each of the three factors on the diversity indices, number of resources foraged and proportion of time-step colonies maintained the number of resources in the nest within predefined bounds.

\section{Results}

\section{Colony performance}

Under all conditions, the performance of colonies rapidly increased, with a stable equilibrium being reached with less that 25 generations of selection (see Electronic Supplementary Material, Fig. S1). After 100 generations, there was considerable variation in performance among the 125 treatments, with the highest value $(3,935)$ being achieved for queens that mated with ten males and had one locus encoding the foraging task response threshold and ten loci encoding the regulatory task response threshold. By contrast, the lowest performance $(1,202)$ occurred for singly mated queens having ten loci for the foraging task and one locus for the regulatory task. The three-way ANOVA (Table 1) revealed that most of the variance $(88.5 \%)$ in colony performance was explained by variation in the number of matings and loci for the foraging task (the interaction between these two variables accounted for another $8.2 \%$ of the variance). By contrast, the number of loci for the regulatory task had only a relatively weak effect on colony performance $(3 \%)$.

Whatever the number of loci for the foraging and regulatory tasks, higher mating number always translated into increased colony performance (one-way ANOVAs, all d.f. $=4, p<0.001$ for the 25 combinations; Fig. 1). This increase was particularly marked when there was only one or a few loci for the foraging task (Fig. 1a and c). Under all conditions, the benefit of each additional mating decreased with increasing mating number.

Colony performance was also always affected by the number of loci for the foraging task (one-way ANOVAs, all d.f. $=4, p<0.001$ for the 25 combinations; Fig. 2). When queens were singly mated, the highest performance was achieved when they had three loci, with performance significantly decreasing with one or more than three loci (Fig. 2a and c). When queens were multiply mated (three to ten matings), the highest performance occurred when there was a single locus for the foraging task and the performance significantly decreased with higher number of loci (Fig. 2b, d).

Table 1 Three-way ANOVA analysis of the effects of number of matings, number of loci for the foraging and regulatory tasks on colony performance

\begin{tabular}{lrrrl}
\hline Factors & $d . f$. & $F$ & $p$-value & $\begin{array}{l}\text { Variance } \\
\text { explained }\end{array}$ \\
\hline No. matings & 4 & $98,317.18$ & $<0.001$ & 0.37 \\
No. loci (foraging task) & 4 & $134,107.87$ & $<0.001$ & 0.51 \\
No. loci (regulatory task) & 4 & $7,781.88$ & $<0.001$ & 0.03 \\
No. matings $\times$ no. loci & 16 & $5,363.11$ & $<0.001$ & 0.08 \\
$\quad$ foraging task) & & & & \\
\hline
\end{tabular}

Only factors and interactions with a significant effect are listed 


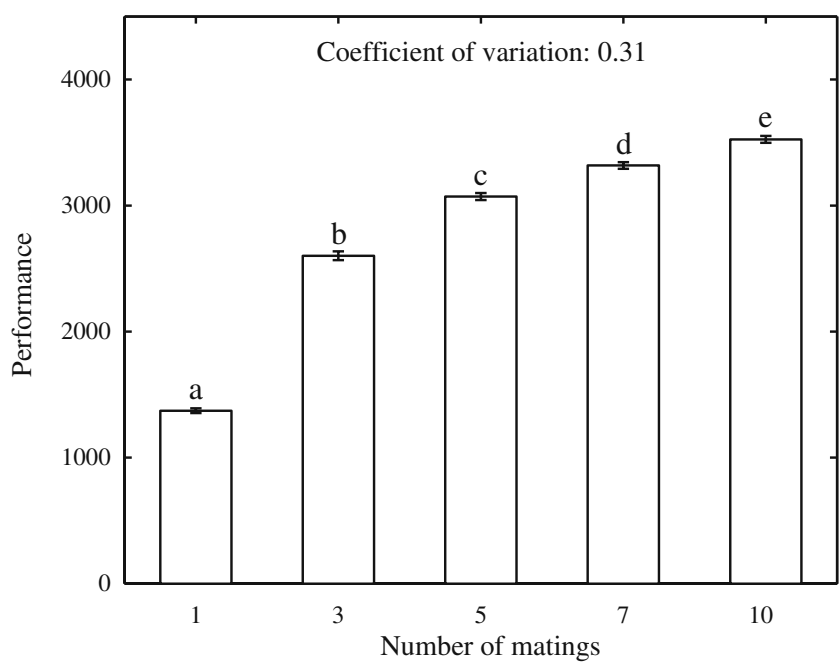

a

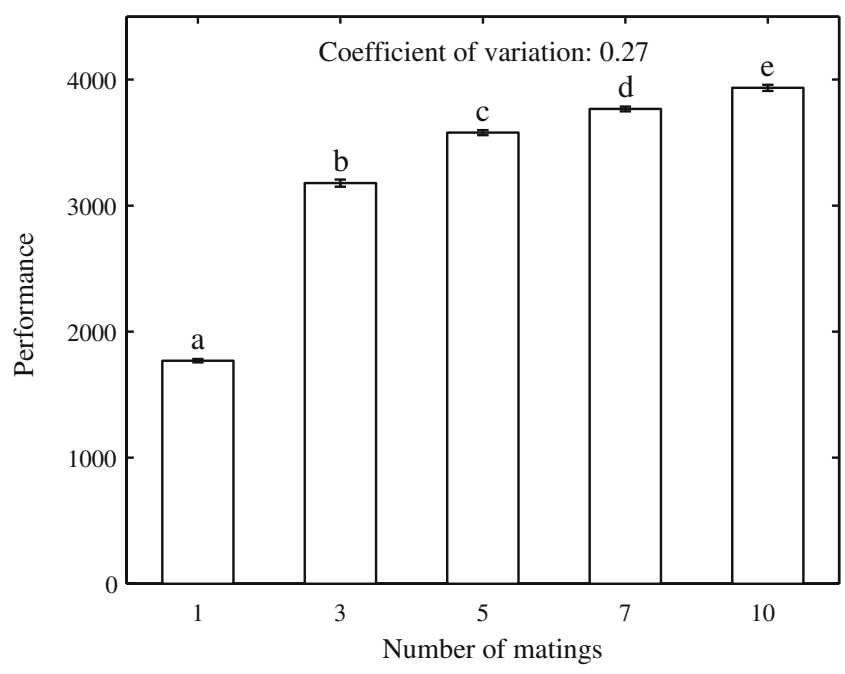

C

Fig. 1 Mean $( \pm S D)$ performance of colonies for different number of matings. Treatments with different letters differed significantly (Tukey-Kramer's multicomparison tests). a One locus (foraging task) and one locus (regulatory task). b Ten loci (forag-

Although the number of loci for the regulatory task also affected colony performance, the effect was relatively small (Fig. 3). In all cases, colony performance increased with a higher number of loci for the regulatory task.

The influence of the number of matings and loci for the foraging and regulatory tasks on the number of workers allocated to the two tasks and the number of workers idle is illustrated in (Electronic Supplementary Material, Fig. S2). Overall, it appears that, in our experimental conditions, most workers engage in the foraging task.

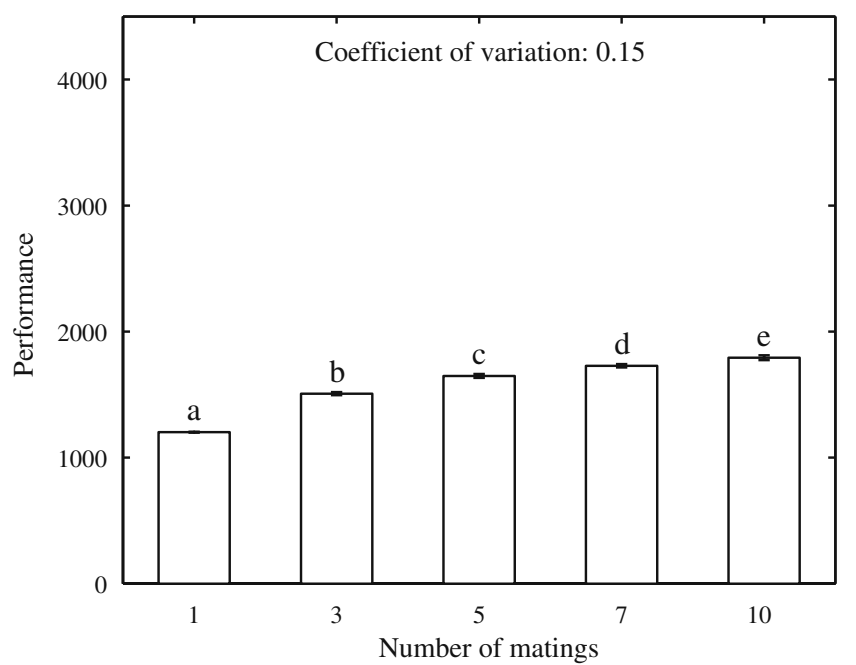

b

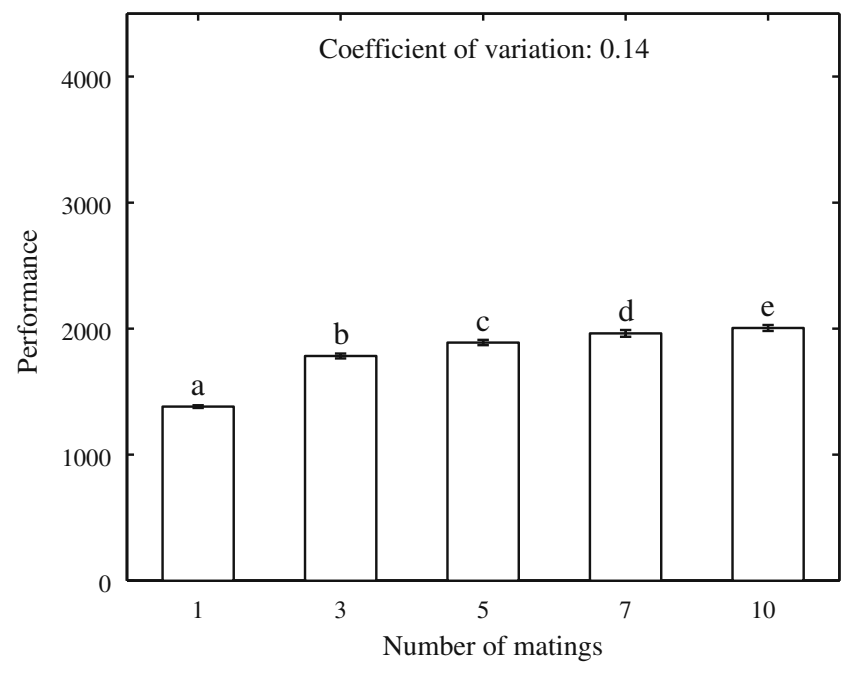

d

ing task) and one locus (regulatory task). c One locus (foraging task) and ten loci (regulatory task). d Ten loci (foraging task) and ten loci (regulatory task)

Behavioural analysis and response threshold diversity

There was extensive variation among treatments $(n=$ $125)$ in the within-colony diversity of response thresholds for the foraging task. The highest diversity (0.27) was when queens mated with ten males and when there was one locus for the foraging and regulatory tasks. By contrast, the lowest diversity (0.04) occurred for singly mated queens with ten loci for the foraging task and one locus for the regulatory task. The withincolony diversity of thresholds for the foraging task was 


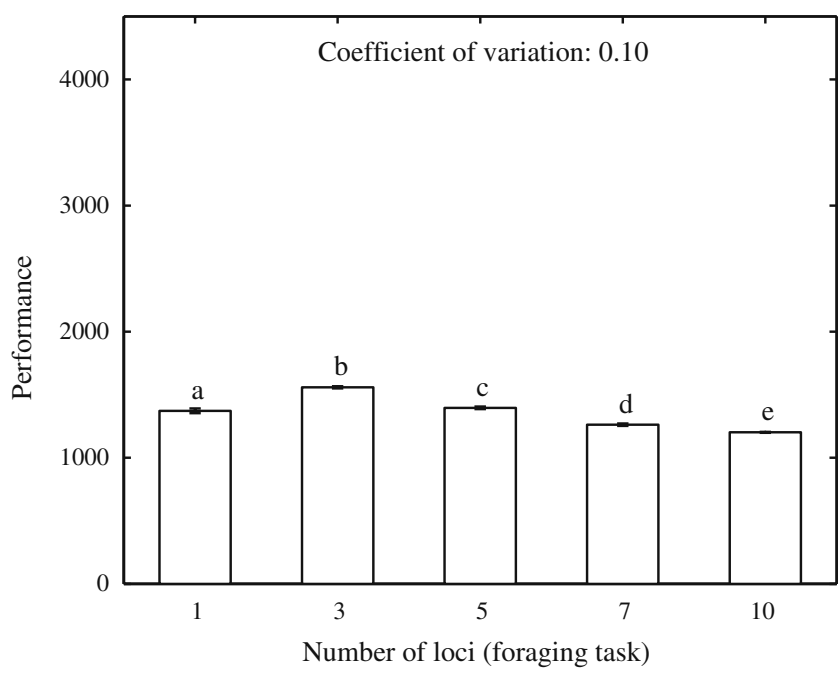

a

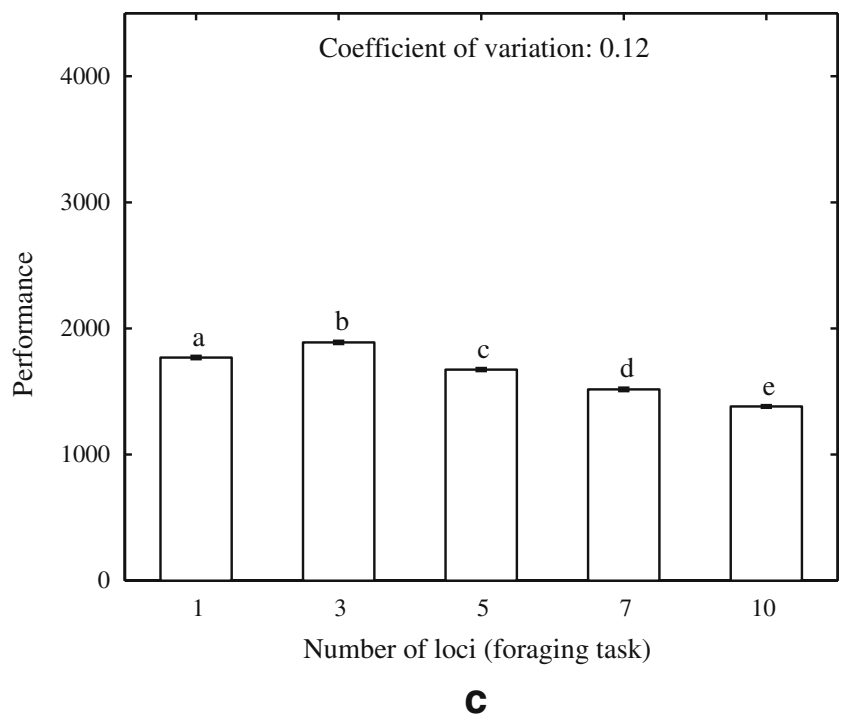

Fig. 2 Mean $( \pm S D)$ performance of colonies for different number of loci encoding the foraging task. Treatments with different letters differed significantly (Tukey-Kramer's multicomparison

strongly correlated with colony performance across the 125 treatments (Table 2). This was because increased threshold diversity for the foraging task was positively associated with the efficiency of both the foraging and regulatory tasks. The three-way ANOVAs revealed that the number of loci for the foraging task, and to a lower extent, the number of matings, were the most important factors affecting the within-colony threshold diversity for the foraging task and the worker efficiency in conducting the foraging task (Electronic Supplementary Material, Table S1; Figs. S3 and S4). The interaction between the number of matings and number of loci for the foraging task also had an effect on the foraging

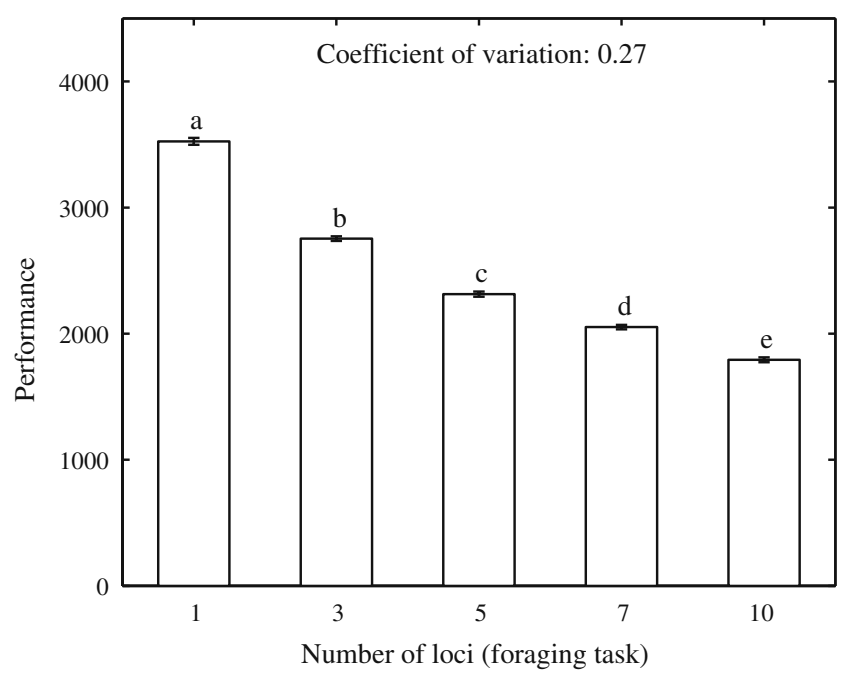

b

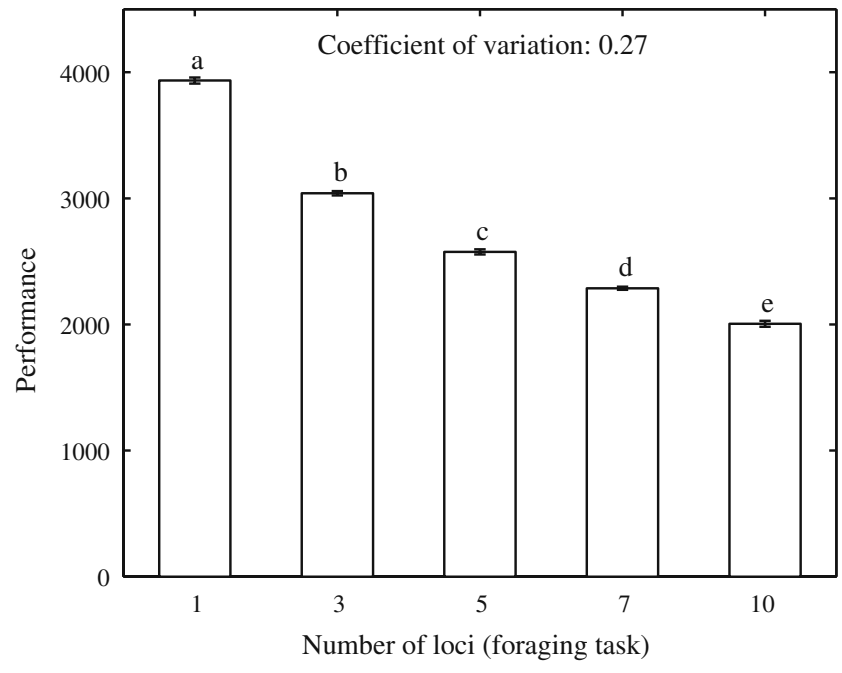

d

tests). a One mating and one locus (regulatory task). b Ten matings and one locus (regulatory task). c One mating and ten loci (regulatory task). d Ten matings and ten loci (regulatory task)

task threshold diversity and efficiency. By contrast, the number of loci for the regulatory task had almost no effect (Electronic Supplementary Material, Table S1; Fig. S5).

There was relatively low variation among treatments $(n=125)$ in the within-colony diversity of response thresholds for the regulatory task. The highest value was when queens mated five times and when there were ten loci for the foraging task and three loci for the regulatory task. By contrast, the lowest diversity occurred for singly mated queens with seven loci for the foraging task and one locus for the regulatory task. Overall, the within-colony threshold diversity for the 


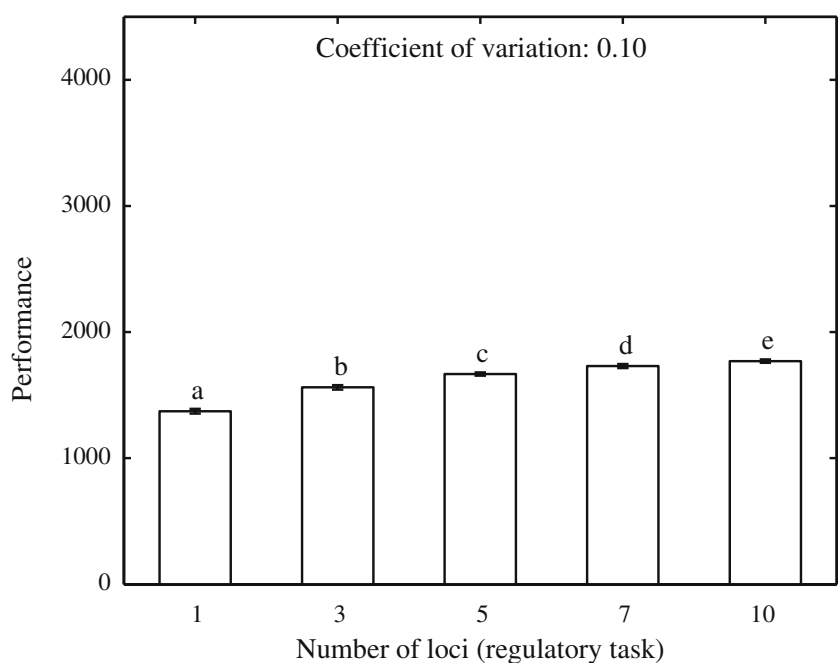

a

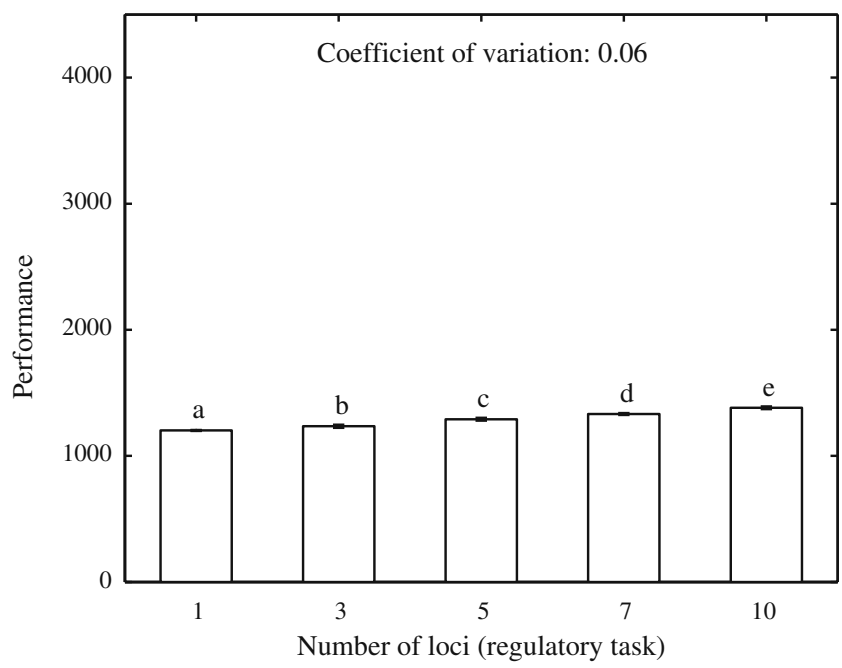

C

Fig. 3 Mean $( \pm \mathrm{SD})$ performance of colonies for different number of loci encoding the regulatory task. Treatments with different letters differed significantly (Tukey-Kramer's multi-

regulatory task was moderately correlated with colony performance (Table 2). This positive correlation was mostly due to the diversity of thresholds for the regulatory task being positively correlated with the efficiency in conducting the regulatory task. The association between the threshold diversity for the regulatory task and the efficiency in conducting the foraging task was also positive, but the correlation was relatively weak. The three-way ANOVAs revealed that most of the variance in threshold diversity and efficiency to conduct the regulatory task was accounted for by variation in the number of matings, which was positively associated with both variables (Electronic Supplementary Material, Table S2; Fig. S6). The number of loci for the

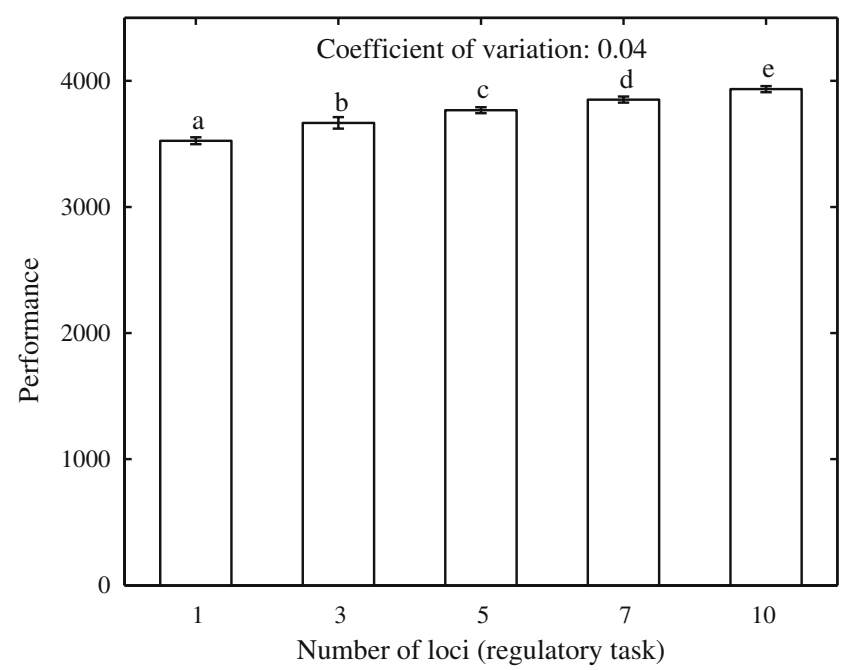

b

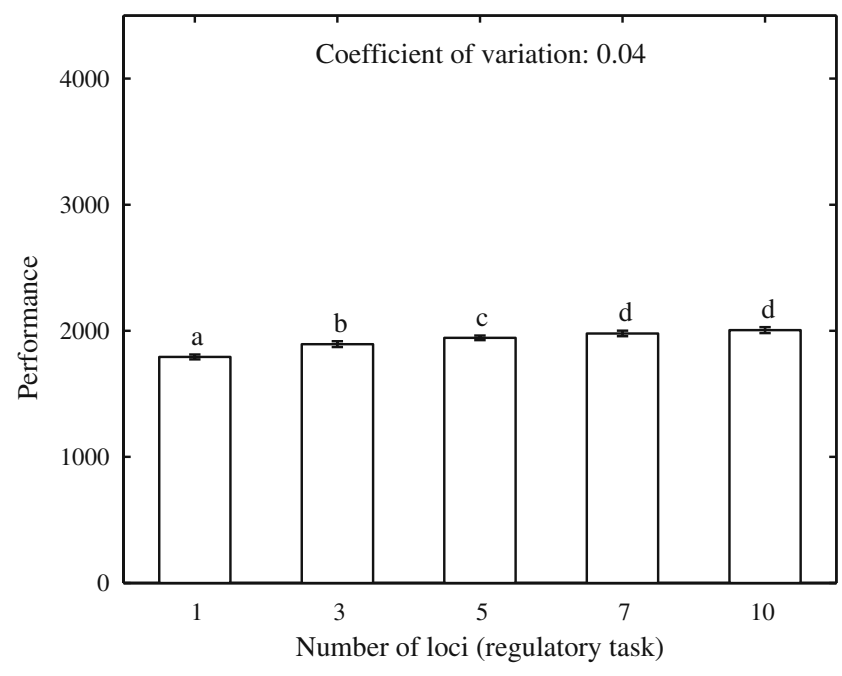

d

comparison tests). a One mating and one locus (foraging task). b Ten matings and one locus (foraging task). c One mating and ten loci (foraging task). d Ten matings and ten loci (foraging task)

foraging and regulatory tasks also had an effect, but it was much lower (Electronic Supplementary Material, Figs. S7 and S8).

Table 2 Correlation between response threshold diversity and (1) colony performance, efficiency of workers in performing the (2) foraging and (3) regulatory task

\begin{tabular}{llll}
\hline & Performance & $\begin{array}{l}\text { Foraging } \\
\text { task efficiency }\end{array}$ & $\begin{array}{l}\text { Regulatory } \\
\text { task efficiency }\end{array}$ \\
\hline $\begin{array}{c}\text { Diversity index } \\
\text { for foraging task }\end{array}$ & $0.58^{*}$ & $0.32^{*}$ & $0.27^{*}$ \\
$\begin{array}{c}\text { Diversity index } \\
\text { for regulatory task }\end{array}$ & $0.11^{*}$ & $0.03^{*}$ & $0.12^{*}$ \\
\hline
\end{tabular}

*Statistically significant at $p=0.05$ level; for all tests $n=625,000$ 
The positive association between within-colony threshold diversity and performance is illustrated by comparing the diversity of response threshold values for the foraging and regulatory tasks in the colonies with the highest, 200th best and 400th best performance (Electronic Supplementary Material, Fig. S9). This comparison reveals that higher mating number translated into a wider range of within-colony response thresholds for both the foraging and regulatory tasks. In addition, the within-colony range of response thresholds for the regulatory task was also positively influenced by the number of loci for the regulatory task. By contrast, the within-colony range of response thresholds for the foraging task was negatively associated with the number of loci encoding this task.

\section{Discussion}

In this study, we investigated how variation in withincolony genetic diversity (due to variation in the number of matings by queens) and genetic architecture underlying the response thresholds for the foraging and regulatory tasks jointly influenced division of labour and performance in simulated colonies of social Hymenoptera. Our simulations revealed significant interactions between the three factors, with colony fitness being greatly influenced by the number of matings and the number of loci for the foraging task. By contrast, the number of loci for the regulatory task only had a small effect.

An increase in the number of matings always translated into higher colony performance. The benefits of any additional mating number were particularly important for low mating frequencies and when there was only one or a few loci encoding the threshold value for the foraging task. The benefits of increased mating number were mediated by an increase in within-colony diversity of response thresholds for both the foraging and regulatory tasks. A possible consequence of the wider range of response thresholds for the foraging task was that workers could forage for the resources under a wider range of task stimuli. In addition, increased mating number may have also benefited the performance of the regulatory task by allowing a more precise control of the number of workers performing the task. As a result, colonies headed by multiply mated queens were more efficient in conducting both the foraging and regulatory tasks. Two theoretical studies had previously investigated how multiple matings by queens influence the ability of workers to regulate nest temperature (Graham et al. 2006; Myerscough and Oldroyd 2004). These studies concluded that, by increasing within- colony genetic variability, polyandry is an important contributor to the ability of colonies to precisely thermoregulate the nest. However, both studies assumed a pre-determined distribution of thresholds within and between patrilines. By allowing the threshold values to evolve by selection, our study extends the results of Myerscough and Oldroyd (2004) and Graham et al. (2006) and demonstrates that an increased number of matings may indeed provide long-term benefits even when the threshold values can evolve adaptively. Consistent with these models, empirical data suggest that higher mating frequency has positive effects on colony fitness (Fuchs and Schade 1994; Mattila and Seeley 2007; Oldroyd et al. 1991) and control of brood nest temperature in the honeybee (Jones et al. 2004, 2007). By contrast, empirical data on the polyandrous ants Cataglyphis cursor and Formica selysi suggest that multiple mating does not translate into more polymorphic workers to facilitate division of labour (Schwander et al. 2005; Fournier et al. 2008). In addition, experimental variation of the colony genetic diversity in the Argentine ant Linepithema humile had no effect on task performance efficiency (Rosset et al. 2005). It is not yet completely clear whether genetic polyethism generally results in more efficient division of labour in colonies that contain more patrilines compared to colonies with fewer patrilines. More work on a wider range of social insect species is needed to determine the influence of multiple matings on division of labour.

The finding that increased number of matings always increases colony performance is in apparent contraction with the observation that the vast majority of ants, social bees, and wasps mate only once (Boomsma and Ratnieks 1996). A possible explanation is that there are also costs to multiple mating (Strassmann 2001), which have not been factored in our simulation. Consequently, it may be beneficial for some species to mate singly. Another explanation on the rarity of multiple matings is that intracolonial genetic diversity may also arise when colonies are headed by multiple queens (polygyny). Consistent with this explanation, empirical data on different species of eusocial Hymenoptera suggests a clear and significant negative relationship between multiple matings and multiple queens (Keller and Reeve 1994; Hughes et al. 2008). More work is needed to determine the relative influence of multiple queens and multiple matings on the efficiency of division of labour.

Colony performance was negatively affected by the number of loci encoding the response threshold for the foraging task. The effect was particularly strong when queens were multiply mated. In all conditions, a higher number of loci for the foraging task translated 
into lower within-colony response threshold diversity for the foraging task and a correlated decrease in foraging efficiency. By contrast, the number of loci for the regulatory task only had a minor effect on colony performance. A higher number of loci for the regulatory task resulted in increased within-colony response threshold diversity for the regulatory task and a greater efficiency to conduct this task.

In an earlier model, Bertram et al. (2003) also investigated how differences in the number of loci encoding the response threshold of pollen foraging influence colony performance. However, they considered only one foraging task and did not conduct selection experiments to determine how allelic values evolve. Our numerical experiments reveal that the influence of genetic architecture depends on the task considered. A system with fewer loci provides the greater fitness return for tasks, such as foraging, which are more efficiently performed when more workers are involved. By contrast, regulatory tasks, such as the regulation of nest temperature or amount of pollen in the nest, are more efficiently conducted when the response thresholds are encoded by many loci. A likely explanation for this difference is that an increased number of loci results in a narrower range of response thresholds because a larger number of allelic values are averaged to determine individual thresholds. The narrower range of response thresholds is beneficial for regulatory tasks (which require precise regulation of worker number), but detrimental for foraging tasks which are most efficiently performed with greater numbers of workers involved. Unfortunately, there is currently only very limited empirical data relating to the genetic architecture of response thresholds. In the honey bee Apis mellifera, it seems that a few major loci (Hunt et al. 1995; Page et al. 2000) have a major effect on individual foraging behaviour. However, more work is needed to determine the number of loci involved and their relative role in determining the task thresholds that stimulate workers to engage in foraging and other tasks.

An important assumption of our model is that individuals used fixed strategies prescribed by their genotype. Of great interest would be to determine whether the occurrence of phenotypic plasticity and noise would affect the results. In particular, it is likely that such plasticity may decrease the benefits of multiple matings on division of labour. However, such a likelihood would be influenced by the various costs associated with phenotypic plasticity (DeWitt et al. 1998), and more work is needed to determine the influence of plasticity.

Another assumption of our model is the manner in which colony fitness was influenced by successful performance of the foraging and regulatory tasks. A colony was awarded fitness for foraging only when the number of food items of the regulatory task was within predefined bounds. Such an allocation of fitness based on absolute bounds is a simplification of conditions observed in nature where the interaction between tasks may be less marked. The use of a less abrupt interaction between the two tasks should lead to similar qualitative results, although the interaction between the number of matings and multiple loci for the regulatory task may be less marked.

In conclusion, our results reveal complex interactions between the effect of the number of matings and the genetic architecture of response thresholds. While an increased number of matings seems to always be beneficial, the best genetic architecture for task thresholds was dependent on the task considered. Moreover, the benefits of an increased number of matings was also strongly dependent on the task performed and the genetic architecture of the response threshold for that task. Unfortunately, there are still only very limited data on the genetic architecture underlying variation in response thresholds in social insects. By explicitly modelling the genetic architecture of response thresholds for individual queens, males and workers and evolving their allelic values, the present study provides a first step toward achieving a better understanding of the mechanisms underlying division of labour and will hopefully elicit more experimental work in this important area of research.

Acknowledgements We thank Markus Waibel for initial explorations, Steffen Wischmann, John Wang, Sara Mitri and Ana Duarte for helpful discussion and comments, two anonymous reviewers for very useful comments, Timothy Stirling for proofreading the manuscript and the Swiss NSF for supporting this work.

\section{References}

Bertram SM, Gorelick R, Fewell JH (2003) Colony response to graded resource changes: an analytical model of the influence of genotype, environment, and dominance. Theor Popul Biol 64:151-162

Bonabeau E, Theraulaz G, Deneubourg JL (1996) Quantitative study of the fixed threshold model for the regulation of division of labour in insect societies. Proc R Soc B 263:1565-1569

Boomsma JJ, Ratnieks FLW (1996) Paternity in eusocial hymenoptera. Phil Trans R Soc B 351:947-975

Calabi P, Traniello JFA (1989) Behavioral flexibility in age castes of the ant Pheidole dentata. J Insect Behav 2:663-677

Cerdá X, Angulo E, Boulay R, Lenoir A (2009) Individual and collective foraging decisions: field study of worker recruitment in the gypsy ant. Behav Ecol Sociobiol 63:551-562

Costa JT, Ross KG (2003) Fitness effects of group merging in a social insect. Proc R Soc B 270:1697-1702

Crosland MWJ, Traniello JFA (1997) Behavioral plasticity in division of labor in the lower termite Reticulitermes fukienensis. Naturwissenschaften 84:208-211 
DeWitt TJ, Sih A, Wilson DS (1998) Costs and limits of phenotypic plasticity. Trends Ecol Evol 13:77-81

Fewell JH, Bertram SM (1999) Division of labor in a dynamic environment: response by honeybees (Apis mellifera) to graded changes in colony pollen stores. Behav Ecol Sociobiol 46:171-179

Fournier D, Battaille G, Timmermans I, Aron S (2008) Genetic diversity, worker size polymorphism and division of labour in the polyandrous ant Cataglyphis cursor. Behav Ecol Sociobiol 75:151-168

Frumhoff PC, Baker J (1988) A genetic component to division of labour within honey bee colonies. Nature 333:358-361

Fuchs S, Schade V (1994) Lower performance in honeybee colonies of uniform paternity. Apidologie 25:155-168

Gordon DM (1989) Dynamics of task switching in harvester ants. Anim Behav 38:194-204

Gordon DM (1991) Behavioral flexibility and the foraging ecology of seed-eating ants. Am Nat 138:379-411

Graham S, Myerscough MR, Jones JC, Oldroyd BP (2006) Modelling the role of intracolonial genetic diversity on regulation of brood temperature in honey bee (Apis mellifera L.) colonies. Insectes Soc 53:226-232

Hölldobler B, Wilson EO (1990) The ants. Belknap, Cambridge

Hughes WOH, Ratnieks FLW, Oldroyd BP (2008) Multiple paternity or multiple queens: two routes to greater intracolonial genetic diversity in the eusocial hymenoptera. J Evol Biol 21:1090-1095

Hunt GJ, Page RE, Fondrk MK, Dullum CJ (1995) Major quantitative trait loci affecting honey bee foraging behavior. Genetics 141:1537-1545

Jeanson R, Fewell JH, Gorelick R, Bertram SM (2007) Emergence of increased division of labor as a function of group size. Behav Ecol Sociobiol 62:289-298

Jones JC, Nanork P, Oldroyd BP (2007) The role of genetic diversity in nest cooling in a wild honey bee, Apis florea. J Comp Physiol A 193:159-165

Jones JC, Myerscough MR, Graham S, Oldroyd BP (2004) Honey bee nest thermoregulation: diversity promotes stability. Science 305:402-404

Julian GE, Cahan S (1999) Undertaking specialization in the desert leaf-cutter ant Acromyrmex versicolor. Anim Behav $58: 437-442$

Karsai I, Wenzel JW (2000) Organization and regulation of nest construction behavior in Metapolybia Wasps. J Insect Behav 13:111-140

Keller L, Reeve H (1994) Genetic variability, queen number, and polyandry in social hymenoptera. Evolution 48:694-704

Lenoir JC, Laloi D, Dechaume-Moncharmont FX, Solignac M, Pham MH (2006) Intra-colonial variation of the sting extension response in the honey bee Apis mellifera. Insectes Soc 53:80-85

Mattila HR, Seeley TD (2007) Genetic diversity in honey bee colonies enhances productivity and fitness. Science 317: 362-364

Mercier J, Lenoir A (1999) Individual flexibility and choice of foraging strategy in Polyrhachis laboriosa F. Smith (hymenoptera, formicidae). Insectes Soc 46:267-272

Myerscough MR, Oldroyd BP (2004) Simulation models of the role of genetic variability in social insect task allocation. Insectes Soc 51:146-152

O'Donnell S (1996) RAPD markers suggest genotypic effects on forager specialization in a eusocial wasp. Behav Ecol Sociobiol 38:83-88
Oldroyd BP, Rinderer TE, Buco SM (1991) Intracolonial variance in honey bee foraging behaviour: the effects of sucrose concentration. J Apic Res 30:137-145

Oldroyd BP, Rinderer TE, Buco SM, Beaman LD (1993) Genetic variance in honey bees for preferred foraging distance. Anim Behav 45:323-332

Oldroyd BP, Sylvester HA, Wongsiri S, Rinderer TE (1994) Task specialization in a wild bee, Apis florea (hymenoptera: apidae), revealed by RFLP banding. Behav Ecol Sociobiol 34:25-30

Page RE, Mitchell SD (1998) Self-organization and the evolution of division of labor. Apidologie 29:171-190

Page RE, Fondrk MK, Hunt GJ, Guzmán-Novoa E, Humphries MA, Nguyen K, Greene AS (2000) Genetic dissection of honeybee (Apis mellifera L.) foraging behavior. J Heredity 91:474-479

Perez-Uribe A, Floreano D, Keller L (2003) Effects of group composition and level of selection in the evolution of cooperation in artificial ants. In: Banzhaf W, Christaller T, Dittrich P, Kim J, Ziegler J (eds) Proceedings of the 7th European conference on artificial life (ECAL'03). Springer, New York, pp 128-137

Ranger S, O'Donnell S (1999) Genotypic effects on forager behavior in the neotropical stingless bee Partamona bilineata (Hymenoptera: meliponidae). Naturwissenschaften 86: 187-190

Robinson GE (1992) Regulation of division of labor in insect societies. Ann Rev Entomol 37:637-665

Rosset H, Keller L, Chapuisat M (2005) Experimental manipulation of colony genetic diversity had no effect on short-term task efficiency in the Argentine ant Linepithema humile. Behav Ecol Sociobiol 58:87-98

Rüppell O, Pankiw T, Page RE (2004) Pleiotropy, epistasis and new QTL: the genetic architecture of honey bee foraging behavior. J Heredity 95:481-491

Schwander T, Rosset H, Chapuisat M (2005) Division of labour and worker size polymorphism in ant colonies: the impact of social and genetic factors. Anim Behav 59:215-221

Seeley TD (1989) Social foraging in honey bees: how nectar foragers assess their colony's nutritional status. Behav Ecol Sociobiol 24:181-199

Seeley TD (1995) The wisdom of the hive. Harvard University Press, Cambridge

Snyder LE (1992) The genetics of social behavior in a polygynous ant. Naturwissenschaften 79:525-527

Sokal RR, Rohlf FJ (1995) Biometry: The principles and practice of statistics in biological research. Freeman, New York

Strassmann J (2001) The rarity of multiple mating by females in the social hymenoptera. Insectes Soc 48:1-13

Trontti K, Thurin N, Sundström L, Aron S (2007) Mating for convenience or genetic diversity? Mating patterns in the polygynous ant Plagiolepis pygmaea. Behav Ecol 18: 298-303

Waibel M, Floreano D, Magnenat M, Keller L (2006) Division of labour and colony efficiency in social insects: effects of interactions between genetic architecture, colony kin structure and rate of perturbations. Proc R Soc B 273:18151823

Wilson EO (1975) Sociobiology: The new synthesis. Belknap, Cambridge

Wilson EO (1984) The relation between caste ratios and division of labor in the ant genus Pheidole (Hymenoptera: formicidae). Behav Ecol Sociobiol 16:89-98 